\title{
Rôle des enzymes protéolytiques du sperme dans la fertilité masculine
}

Il existe, dans les spermatozoïdes et dans les sécrétions des glandes sexuelles accessoires, de nombreuses enzymes protéolytiques qui interviennent dans la maturation du spermatozoïde, la fluidification de l'éjaculat, l'augmentation de la mobilité des spermatozoïdes et l'hydrolyse du mucus cervical. L'acrosine, enfin, intervient dans la pénétration du spermatozoïde à travers la membrane pellucide de l'ovule et joue un rôle clé dans le pouvoir fécondant des gamètes mâles. Plusieurs types d'infertilités masculines semblent secondaires à des anomalies de ces enzymes protéolytiques, par exemple un défaut de l'antigène prostatique entraînant une liquéfaction anormale de l'éjaculat ou une diminution de l'acrosine perturbant gravement le pouvoir fécondant des spermatozoïdes.

\section{Jean Y. Dubé \\ Professeur titulaire à l'univer- sité Laval}

\section{Roland R. Tremblay \\ Professeur titulaire à l'univer- sité Laval}

\section{RÉFÉRENCES}

1. Zaneveld LJD, Polakoski KL, Schumacher GFB. The proteolytic enzyme systems of mammalian genital tract secretions and spermatozoa. In : Reich E, Rifkin DB, Shaw E, eds. Proteases and Biological Control. Cold Spring Harbor Conferences on Cell Proliferation. Cold Spring Harbor : Cold Spring Harbor Laboratory, 1975 : 683-706.

\section{ADRESSE}

J.-Y. Dubé, R.R. Tremblay: laboratoire de régulation hormonale, centre hospitalier de l'université Laval, 2705, boulevard Laurier,

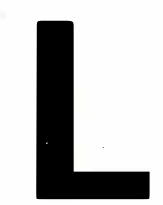

es enzymes protéolytiques sont présentes dans toutes les cellules de l'organisme et dans tous les liquides biologiques. Ces enzymes contribuent au déroulement de processus aussi divers que la digestion des aliments, la coagulation sanguine et la dégradation intracellulaire des protéines. Dans les organes reproducteurs mâles et, plus particulièrement, dans les sécrétions qui y sont produites, on les retrouve en concentration élevée [1]. Les caractéristiques physico-chimiques et enzymatiques de ces proteases sont décrites dans le Tableau I. Leur présence est connue depuis plusieurs décennies, mais on ne fait que commencer à entrevoir plus clairement leur action précise depuis le moment où les spermatozoïdes pénètrent dans l'épididyme jusqu'à celui où ils vont fertiliser l'œuf dans la trompe utérine. Entre ces deux temps, les spermatozoïdes auront été mélangés avec les abondantes sécrétions des deux principales glandes accessoires de l'appareil reproducteur de l'homme, la prostate et les vésicules séminales. Nous voudrions montrer ici, à l'aide de données récentes, que l'action de ces enzymes protéolytiques peut être importante pour la fertilité masculine.

\section{Maturation épididymaire des spermatozoïdes}

Quand les spermatozoïdes émergent des testicules, ils apparaissent complètement différenciés à l'examen microscopique, à l'exception d'une gouttelette cytoplasmique qu'ils contiennent et qui n'est pas présente dans le spermatozoïde éjaculé. Ils ont cependant besoin d'une maturation supplémentaire pour acquérir leur motilité normale et leur pouvoir fécondant. Cette maturation se fait progressivement au cours du transit dans l'épididyme et elle s'achève au niveau de sa 
Tableau 1

CARACTERISTIQUES PHYSICOCHIMIQUES ET ENZYMATIQUES DES PROTÉASES MAJEURES DE L'APPAREIL REPRODUCTEUR DE L'HOMME

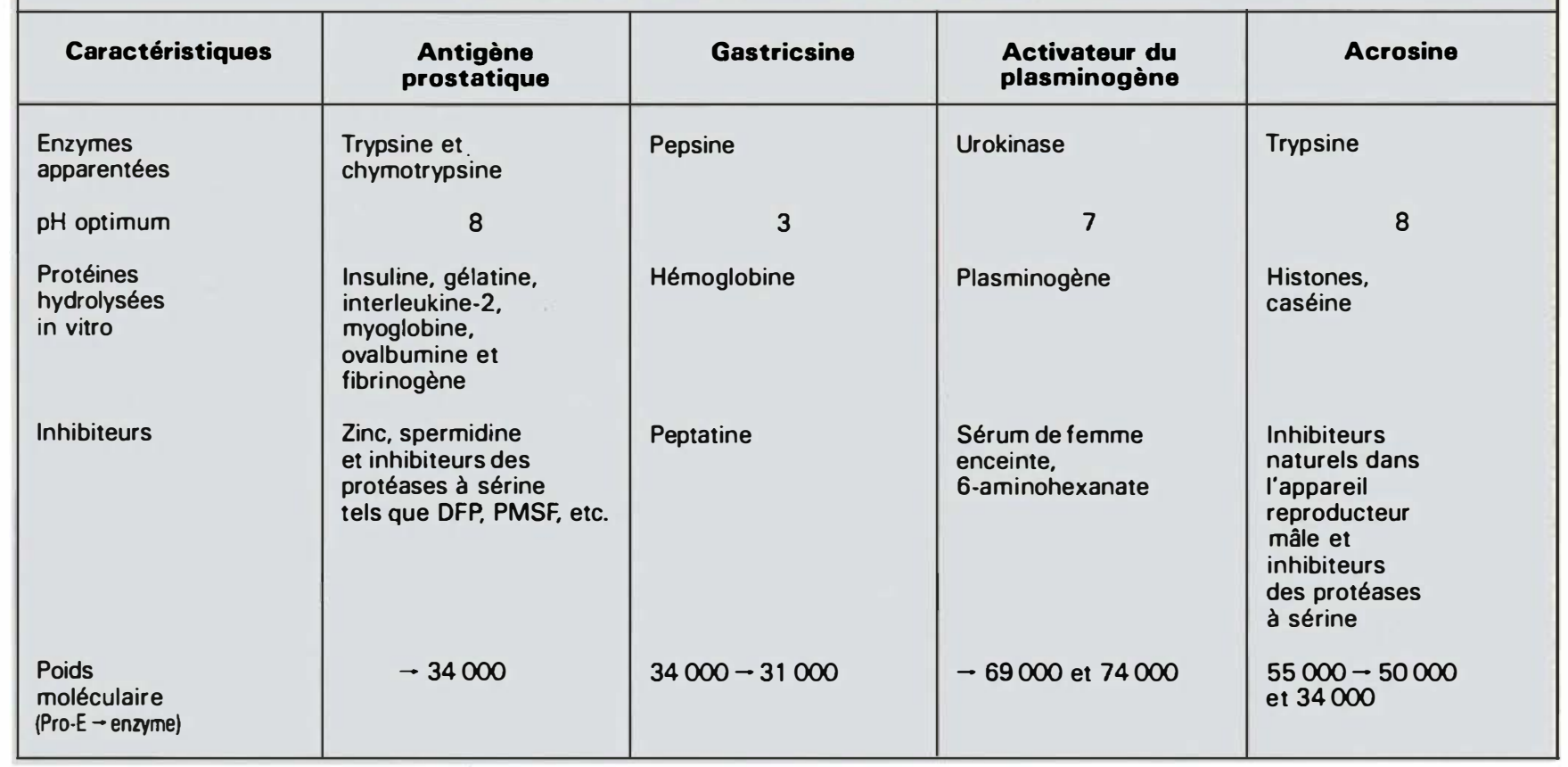

DFP: diisopropyl-fluorophosphate; PMSF : fluorure de phénylméthylsulfonyl; Pro-E = proenzyme.

partie caudale [2]. D'un point de vue moléculaire, on ne sait pas exactement ce que le terme maturation signifie. Toutefois, si l'on examine par électrophorèse la composition des protéines de surface du spermatozoïde, on peut noter que des changements importants se sont produits au cours du transit épididymaire. On observe d'abord, au niveau de la tête de l'épididyme, une disparition rapide de toutes les principales protéines de surface du spermatozoïde testiculaire et l'apparition de nouvelles protéines, dont certaines proviennent des sécrétions épididymaires [3]. D'autres modifications du même ordre surviennent dans les régions médianes et caudales de l'épididyme. On a montré, au moins dans le cas du spermatozoïde de bélier, que la disparition des protéines de surface suppose leur pénétration dans la cellule et leur $\mathrm{m} / \mathrm{s} n^{\circ} 2$ vol. 4, féurier 88 dégradation protéolytique [4]. De plus, même si la chose n'a jamais été parfaitement vérifiée, on peut aussi envisager que des protéases du liquide épididymaire puissent hydrolyser totalement ou partiellement certaines protéines de la membrane plasmique du spermatozoïde. Jusqu'à maintenant, on dispose de très peu d'informations sur la signification physiologique de ces phénomènes et on ne peut que présumer qu'ils sont associés à certaines des activités cellulaires des surfaces membranaires comme la communication entre cellules, leur adhésivité, leur motilité, leur pouvoirde transformation et de différenciation. Les enzymes protéolytiques impliquées dans le processus de dégradation des protéines de surface n'ont pas encore été isolées ni caractérisées. Des glycoprotéines de surface peuvent aussi être modifiées au niveau de leur cupule glucidique, ce qui pourrait aussi avoir des conséquences fonctionnelles sur le spermatozoïde.

\section{Les protéases de la sécrétion prostatique}

La prostate de l'homme sécrète plusieurs enzymes protéolytiques différentes, dont les propriétes et les rôles commencent à être mieux connus. Ces protéases sont d'abord l'antigène prostatique [5], dont les caractéristiques semblent identiques à celles de la protéase neutre, encore appelée séminine [6]; ensuite, la protéase acide, aussi désignée sous les noms de pepsinogène [7] et de gastricsinogene [8]; enfin, les activateurs du plasminogène [9]. L'antigène prostatique est utilisé depuis quelques années comme marqueur du cancer de la prostate au même titre que la phosphatase 


\section{REFEERENCES}

2. Hamilton DW. Suructure and function of the epithelium lining the ductus efferentes, ductus epididymis and ductus deferens in the rat. In : Hamilton DW, Greep RO, eds. Handbook of Physiology, Male Reproductive System, sect 7, Endocrinology, vol 5. Bethesda : American Physiological Society, 1975 :259301.

3. Dacheux JL, Voglmayr JK. Sequence of sperm cell surface differentiation and its relationship to exogenous fluid proteins in the ram epididymis. Biol Reprod 1983 ; 29 : 103346.

4. Voglmayr JK, Fairbanks B, Vespa DB, Colella JR. Studies on mechanisms of surface modification in ram spermatozoa during the final stages of differentiations. Biol Reprod $1982 ; 26: 483-500$

5. Wang MC, Valenzuela LA, Murphy GP, Chu TM. Purification of a human prostate specific antigen. Invest Urol 1979 ; 17 : 159-63.

6. Dubé JY, Lazure C, Tremblay RR. Dog prostate arginine esterase is related to human prostate specific antigen. Clin Invest Med $1986 ; 9$ : 51.4 .

7. Lundquist F, Seedorf HH. Pepsinogen in human seminal fluid. Nature 1952; 170 : 1115-6.

8. Chiang L, Contreras L, Chiang J, Ward PH. Human prostatic gastricsinogen: the precursor of seminal fluid acid proteinase. Arch Biochem Biophys 1981 ; 210 : 14-20.

9. Propping D, Tauber PF, Zaneveld LJD, Schumacher GFB. Purification of plasminogen activators from human seminal plasma. Biochem J 1978; 171 : 435-44.

10. Kuriyama M, Wang MC, Papsidero LD, et al. Quantitation of prostate specific antigen in serum by a sensitive enzyme immunoassay. Cancer Res 1980 ; 40 : 4658-62.

11. Watt KWK, Lee PJ, M'Timkulu T, Chan WP, Loor R. Human prostate-specific antigen: structural and functional similarity with serine proteases. Proc Natl Acad Sci USA $1986 ; 83$ : $3166-70$

12. Chapdelaine P, Dubé JY, Frenette G, Tremblay RR. Identification of arginine esterase as the major androgen-dependent protein secreted by dog prostate and preliminary molecular characterization in seminal plasma. J Androl 1984 ; 5 : 206-10.

13. Lilja H. A kallikrein-like serine protease in prostatic fluid cleaves the predominant seminal vesicle protein. J Clin Invest 1985 ; 76 : 1899-903.

14. Symer F, Moghissi K, Yanez J. Isolation of a factor from normal human semen that accelerates dissolution of abnormally liquefying semen. Fertil Steril 1975 ; 26 : 1064-9.

15. Chong A, Cappiello W, Weinrieb S. The effects of kinins (kallikrein) on recovery of motility of frozen human spermatozoa. Fertil acide prostatique[10]. Avec la mise en évidence de la séquence complète de cette protéine en 1986 [11], on sait maintenant que c'est une protéase à sérine avec une spécificité semblable à celle de la chymotrypsine et de la trypsine. De plus, elle présente une très grande homologie avec plusieurs enzymes de la famille des kallikréines et avec une arginine estérase sécrétée par la prostate du chien [12]. Le substrat endogène de cette enzyme pourrait être la protéine structurale prédominante du coagulum formé lors de l'éjaculation [13]. Cette protéine basique, de poids moléculaire élevé, provient des vésicules séminales. Il est intéressant de noter que quelques sujets hypofertiles présentent une liquéfaction anormale de leur éjaculat, ce qui conserve les spermatozoïdes dans un état d'immobilisation. Lorsque de l'antigène prostatique purifié est ajouté au coagulum de ces sujets, la vitesse de liquéfaction est grandement accélérée in vitro [14].

Une autre propriété des protéases de la famille des kallikréines est leur capacité d'hydrolyser les kininogenes avec libération des kinines vasoactives comme la kallidine et la bradykinine. Certaines publications [15] suggèrent que cette libération de kinine contribue à augmenter la mobilité des spermatozoïdes, laquelle est une condition préalable à leur pénétration du mucus cervical. On a montré également que la protéase neutre du plasma séminal (ou l'antigène prostatique) est capable de libérer des kinines à partir du kininogène in vitro[16]. De plus, un fragment de la protéine structurale des vésicules séminales humaines présente une homologie de séquence de $47 \%$ avec une portion d'un kininogène des bovins [17]. Il serait donc possible que cette protéine structurale du coagulum séminal puisse former des kinines sous l'action de l'antigène prostatique et activer la mobilité des spermatozoïdes.

Les kallikréines glandulaires. semblent également impliquées dans la régulation du flot san- guin local des organes qui ont une activité sécrétrice importante comme le pancréas, les glandes salivaires et le rein [18]. Afin que cette activité se manifeste, il faut que la kallikréine diffuse dans les espaces interstitiels et les vaisseaux pour engendrer, localement, des kinines à partir du kininogène. L'accroissement du débit sanguin prostatique par les kinines pourrait être particulièrement important lors de l'excitation sexuelle et augmenterait le volume de liquide sécrété. Bien qu'il ait été mis en évidence que la protéase neutre du plasma séminal puisse augmenter la perméabilité vasculaire lorsqu'elle est injectée dans la peau du lapin, il reste à prouver que ce mécanisme se déroule au niveau prostatique.

On peut également envisager plusieurs autres rôles pour l'antigène prostatique, notamment l'hydrolyse spécifique de certaines protéines de membrane du spermatozoïde, ou encore des protéines du mucus cervical, ce qui accélérerait la pénétration des spermatozoïdes dans ce fluide. Il a aussi été suggéré que ces protéines pourraient induire, grâce à la formation de kinines, des contractions de l'utérus et des trompes [16].

Les autres enzymes protéolytiques qui proviennent de la prostate sont la protéinase acide et des activateurs du plasminogène dont les rôles sont moins bien connus $[7,8]$. La protéinase acide est sécrétée sous la forme d'un zymogène inactif, le gastricsinogène. Étant donné que l'activation de cette pro-enzyme se fait à un $\mathrm{pH}$ acide, l'action de l'enzyme ne se manifeste sûrement pas avant l'éjaculation puisque le $\mathrm{pH}$ du plasma séminal est d'environ 7,4. En revanche, dans le vagin, où le $\mathrm{pH}$ peut s'abaisser jusqu'à 4 , il est logique de penser que l'enzyme pourrait être impliquée dans la digestion des mucoprotéines.

Quant aux deux activateurs du plasminogène qui ont été isolés du plasma séminal [9] et qui sont plus concentrés dans la fraction 


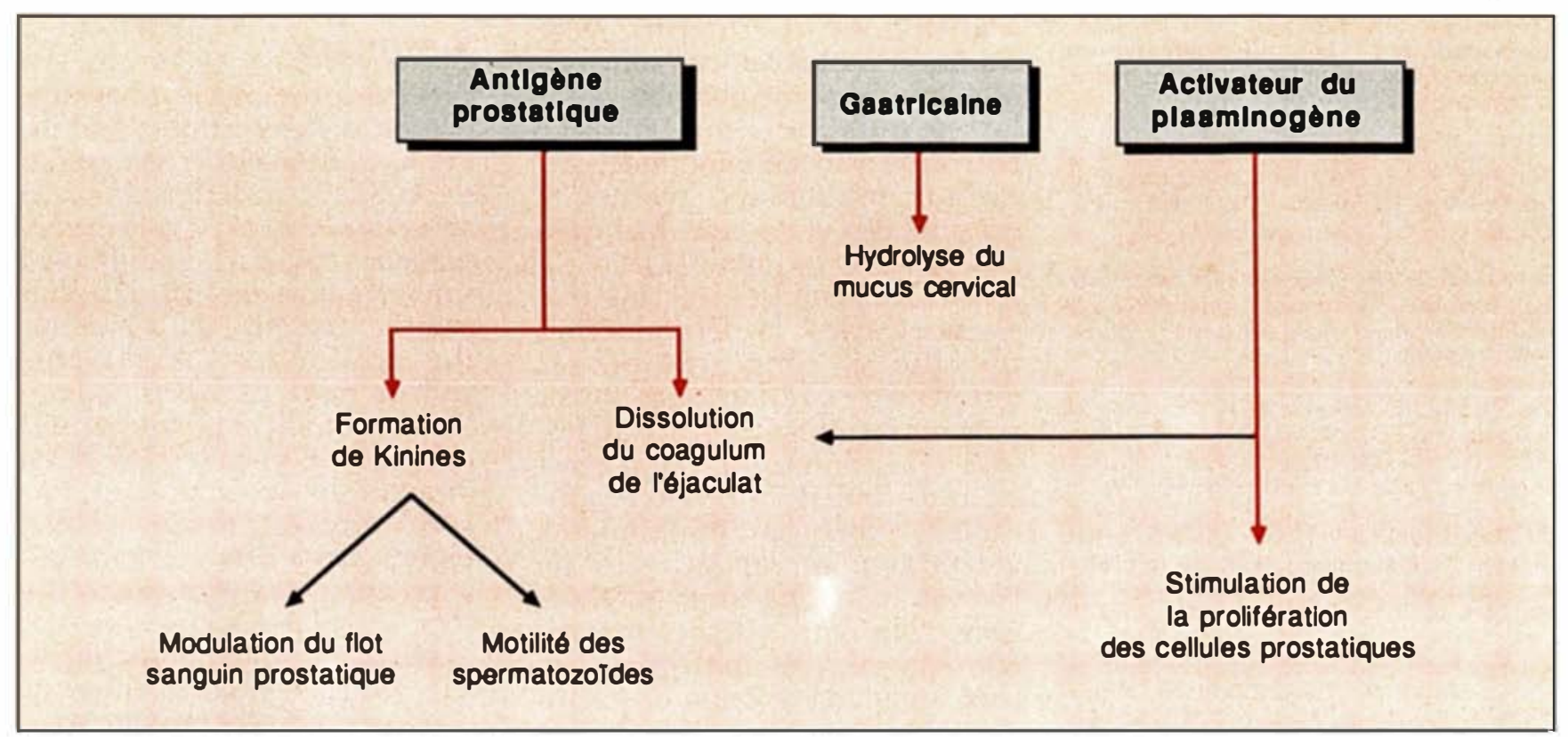

Figure 1. Rôles probables des trois protéases majeures sécrétées par la prostate humaine, c'est-à-dire I'antigène prostatique, la gastricsine et l'activateur du plasminogène.

prostatique de l'éjaculat [19], leurs substrats habituels, c'est-àdire le plasminogène, la prothrombine, le fibrinogène et le facteur XIII, sont absents du plasma séminal. A ce jour, les seuls rôles proposés pour ces activateurs du plasminogène au niveau de l'éjaculat seraient une action coordonnée avec les autres enzymes protéolytiques du plasma séminal dans la dissolution du coagulum. On peut cependant envisager d'autres rôles au niveau de la prostate ellemême, puisque les activateurs du plasminogène semblent être particulièrement présents dans les tissus où la prolifération et la croissance sont élevées. Par exemple, dans les tumeurs expérimentales de la prostate chez les rats $\mathrm{Nb}$, l'activité enzymatique de l'activateur du plasminogène est très élevée [20].

Les rôles présumés des trois protéases principales de la sécrétion prostatique sont résumés dans la figure1. Quant aux autres glandes accessoires, comme les vésicules séminales et les glandes bulbo-urétrales, la nature de leurs produits de sécrétion n'a pas encore fait l'objet d'analyses $\mathrm{m} / \mathrm{s} n^{\circ} 2$ vol. 4, feurier 88 poussées. Mais on sait qu'elles peuvent aussi contribuer à la sécrétion d'une certaine quantité d'activateurs du plasminogène et de protéases acides qui se retrouvent éventuellement dans le plasma séminal.

\section{Le système \\ proacrosine-acrosine}

L'une des enzymes protéolytiques qui occupe une position clé dans la fertilité masculine est certainement l'acrosine du spermatozoïde. La littérature scientifique est cependant assez déroutante autant sur les formes moléculaires de la proacrosine et de ses sousproduits d'activation que sur son rôle physiologique dans différentes espèces animales [21]. Les divergences notées quant au poids moléculaire sont dues au fait qu'une fois activée, l'acrosine est convertie par autodigestion en fragments plus petits. De plus, comme cette protéine est présente en faibles quantités dans les spermatozoïdes et qu'elle est assez labile à la congélation, on peut comprendre aisément les difficultés auxquelles sont confrontés les biochimistes. De plus, on ne pos- sède pas encore d'inhibiteurs véritablement spécifiques permettant d'étudier son rôle biologique. Malgré tout, on peut affirmer que la proacrosine est biosynthétisée sous la forme d'un peptide de poids moléculaire supérieur à 50000 et que l'autolyse de l'acrosine donne naissance à plusieurs peptides dont l'un, de $34 \mathrm{kDa}^{*}$, qui a encore toute son activité enzymatique, est plus stable et a été observé par plusieurs groupes de chercheurs. Les seules données que nous possédons sur la structure primaire de l'acrosine ont été obtenues par l'étude de l'enzyme du verrat, où l'on a déterminé la séquence des 50 premiers acides aminés de la portion $\mathrm{NH}_{2}$ terminale de la molécule [22]. Ces résultats montrent que la protéine a une assez grande homologie avec les protéases à sérine et, en particulier, avec la plasmine humaine et la chymotrypsine bovine. Toutefois, l'acrosine se démarque des autres protéases à sérine par son poids moléculaire, qui est probablement deux fois plus élevé que celui des autres enzymes de cette famille. De plus,

* kDa: kilodalton. 


\section{RÉFÉRENCES}

16. Suominen J. The purification and new properties of the neutral proteinase in human semen. Int J Fertil 1974; 19 : 121-8.

17. Lilja H, Jeppson JO. Amino acid sequence of the predominant basic protein in human seminal plasma. FEBS Lett 1985; $182: 181-4$.

18. Orstavik TB, Carretero OA, Scicli AG. Kallikrein-kinin system in regulation of submandibular gland blood flow. Am J Physiol 1982 ; 242 : 1010-4.

19. Tauber PF, Zaneveld LJD, Propping D, Schumacher FGB. Components of human split ejaculates. II. Enzymes and proteinase inhibitors. J Reprod Fertil 1976 ; 46 : 165-71.

20. Rennie PS, Bruchovsky N, Noble RL, Shudon M. Plasminogen activator in prostatic tumors of $\mathrm{Nb}$ rats. Biochim Acta 1980 . 632: $437-43$

21. Polakoski KL, Siegel MS. The proacrosin-acrosin system. In : Paulson JD, NegroVilar A, Lucena E, Martini L, eds. Andrology: Male Fertility and Sterility. New York : Academic Press Inc, 1986 : 359-75.

22. Fock-Nüzel R, Lottspeich F, Henschen A, Müller-Esterl W, Fritz H. N-terminal amino acid sequence of boar sperm acrosin. Homology with other serine proteinase. HoppeSeyler's Z. Physiol Chem 1980; 361 : 1823-8.

23. Johnson LA, Garner DL, Truitt-Gilbert A J, Lessley BA. Immunocytochemical localization of acrosin on both acrosomal membranes and in the acrosomal matrix of porcine spermatozoa. J Androl 1983 ; 4 : 222-9.

24. Van der Ven HH, Kennedy WP, Kaminski JM, Jeyendran RS, Zaneveld LJD. Human sperm acrosin as a fertility marker. 12th Annual Meeting of the American Society of Andrology (abstr 14). Philadelphie: JB Lippincott Company, 1987.

25. Schill WB. Quantitative determination of acrosin in human spermatozoa. Fertil Steril $1974 ; 25$ : 703-12.

26. Mohsenian M, Syner FN, Moghissi KS. A study of sperm acrosin in patients with unexplained infertility. Fertil Steril 1982 ; 37 : 2239 .

27. Kallajoki M, Parvinen M, Suominen JJO. Expression of acrosin during mouse spermatogenesis: a biochemical and immunocytochemical analysis by a monoclonal antibody CllH. Biol Reprod 1986; 35 : 157-65.

28. Elce JS, Graham EJ, Zboril G. Monoclonal antibodies to bovine and human acrosin. Biochem Cell Biol 1986; 64 : 1242-8.

\section{TIRÉS A PART}

J.-Y. Dubé : laboratoire de régulation hormonale, centre hospitalier de l'université Laval, 2705, boulevard Laurier, Sainte-Foy, Québec, la protéine est beaucoup plus hydrophobe. Cette propriété explique probablement son affinité pour les membranes cellulaires puisque l'on retrouve l'acrosine associée en grande partie aux membranes internes et externes de l'acrosome ainsi qu'à la matrice acrosomiale [23].

L'acrosine semble avoir un rôle essentiel dans la reproduction puisque sa concentration est directement proportionnelle au pouvoir fécondant des spermatozoïdes [24]. Son site d'action exact est plus difficile à préciser puisqu'il n'existe pas d'inhibiteurs véritablement spécifiques de l'acrosine. Malgré tout, les expériences in vitro suggèrent que cette enzyme participe le plus probablement à la réaction de l'acrosome, c'est-à-dire à la vésiculation et à la dispersion de la membrane de la tête du spermatozoïde et permet la liaison du spermatozoïde à la zone pellucide, dans laquelle celui-ci se fraie un canal étroit par digestion. Toutes ces actions sont impossibles pour les spermatozoïdes à tête ronde, qui sont génétiquement dépourvus d'acrosome et d'acrosine [25]. De plus, chez les sujets présentant une oligozoospermie, de même que chez certains de ceux dont l'infertilité était inexpliquée, on a mis en évidence, par une épreuve radioimmunologique, une diminution de l'acrosine dans le sperme [26]. En plus de ces déficiences partielles, on peut penser que certains sujets pourraient avoir d'autres types d'anomalies, notamment des défauts d'activation de la proacrosine ou encore des quantités élevées d'inhibiteurs. En conclusion, on ne fait que commencer à entrevoir un peu plus clairement le rôle diversifié que jouent les enzymes protéolytiques dans la fertilité masculine. Dans un avenir prochain, les biochimistes connaîtront sans doute encore mieux les détails de cette action, en particulier celle de l'acrosine, grâce aux anticorps monoclonaux dirigés contre cette protéine qui sont maintenant disponibles $[27,28]$

\section{Summary}

Several proteolytic enzymes present on spermatozoa and in the various accessory sex gland secretions contribute to the fertilizing ability of male gametes. In the epididymis, uncharacterized proteolytic enzymes coming either from the epididymal secretions and/or from the spermatozoa are able to alter sperm coating proteins, a process which is thought to be important for spermatozoa maturation. Upon ejaculation, the spermatozoa are mixed with the accessory sex gland secretions and in particular those of the prostate which contain 3 different proteases, namely prostate specific antigen, gastricsinogen and plasminogen activators. These enzymes are probably involved in the dissolution of the coagulum formed during ejaculation, in the increase of spermatozoa mobility, and in the hydrolysis of sperm-coating proteins and of cervical mucus proteins. Finally, one of the key enzyme for the fertilizing ability of spermatozoa is acrosin whose main roles include a participation in the acrosomal reaction of the spermatozoa and in the digestion of a path through the zona pellucida by the sperm head. The importance of the proteolytic enzyme system for male reproduction is evidenced by the fact that specific alterations of at least some components of this system are associated with male infertility.

\section{Remerciements}

Les auteurs expriment leur gratitude au professeur Jacques Boulay, chef du service d'hématologie de l'hôpital Saint-François-d'Assise, pour son aide à la rédaction finale de leur texte. Ils tiennent également à souligner la collaboration de Mme Lucie Turcotte dans la préparation de cet article. 